\title{
Caracterización del Sector Productivo Local Asociado a el Programa de Tecnología en Contabilidad Sistematizada: Revisión Teórica
}

\section{Characterization of the Local Productive Sector Associated with the Systematized Accounting Technology Program: Theoretical Review}

\author{
Jhorquis Machado-Licona* \\ Corporación Universitaria Rafael Núñez \\ ORCID iD: https://orcid.org/0000-0002-6987-7658 \\ Jhorquis.machado@curnvirtual.edu.co \\ Liliana D. C. Franco-Blanco \\ Corporación Universitaria Rafael Núñez \\ ORCID iD: https://orcid.org/0000-0003-0490-1079 \\ Liliana.franco@curnvirtual.edu.co
}

\footnotetext{
* Autor a quien debe ser dirigida la correspondencia
}

Fecha de recepción: 04/03/2021

Fecha de evaluación: 31/03/2021

Fecha de aceptación: 07/06/2021

Cómo citar: Machado-Licona, J., \& Franco-Blanco, L. (2021). Caracterización del sector productivo local asociado a el programa de tecnología en contabilidad sistematizada: revisión teórica. Revista Cientifica Anfibios, 4(1). https://doi.org/10.37979/afb.2021v4n1.85

\section{Resumen}

Atribución-NoComercial-CompartirIgual 4.0 Internacional

El crecimiento económico y social de las naciones, responde al desarrollo de competencias de su población y de cómo se integran esas competencias al sector productivo, es por ello que debe ser relevante para los programas técnicos y profesionales desarrollar estudios que permitan la caracterización el sector empresarial, logrando así identificar los requerimientos presentes y las tendencias futuras en relación con los perfiles que deben poseer sus egresados. Esa anticipación impulsa a las instituciones de educación superior y las lleva a ubicarse en los principales lugares a nivel nacional e internacional. A raíz de lo anterior el presente proyecto estudio desde el programa de tecnología en contabilidad sistematizada, tiene como objetivo general caracterizar el sector productivo de Cartagena de Indias con la finalidad de identificar las necesidades o requerimientos relacionados con el perfil del tecnólogo en contabilidad sistematizada. El enfoque implementado en la investigación es de naturaleza cualitativo, en una primera etapa se realiza la revisión documental con el propósito de sentar las bases para la identificación de las variables a considerar en la segunda etapa de recolección y procesamiento de datos. Cabe aclara que los resultados mostrados en el presente documento derivan de la primera fase de la investigación.

\section{Palabras clave}

Sector productivo; programa de tecnología en contabilidad sistematizada; crecimiento económico; desarrollo social; competencias

\begin{abstract}
The economic and social growth of nations responds to the development of competencies of its population and how these competencies are integrated into the productive sector, which is why it should be relevant for technical and professional programs to develop studies that allow the characterization of the business sector, thus identifying the present requirements and future trends in relation to the profiles that their graduates should have. This anticipation drives higher education institutions and leads them to position themselves in the main national and international places. As a result of the above, the general objective of the present study project from the program of technology in systematized accounting is to characterize the productive sector of Cartagena de Indias with the purpose of identifying the needs or requirements related to the profile of the technologist in systematized
\end{abstract}


accounting. The approach implemented in the research is qualitative in nature, in a first stage the documentary review is carried out with the purpose of laying the foundations for the identification of the variables to be considered in the second stage of data collection and processing. It should be clarified that the results shown in this document derive from the first phase of the research.

\section{Keywords}

Productive sector; systematized accounting technology program; economic growth; social development; competencies

\section{Introducción}

La población es el elemento garante del empuje económico de las naciones de allí la importancia dada al capital humano, ahora bien, cabe aclarar que no solo es la población es la "población bien preparada", esto se interpreta como el capital humano con competencias deseadas por el sector productivo, quiere decir que se trata de población con las competencias adecuadas para cubrir las principales necesidades de los sectores que impulsan el desarrollo de las regiones. El desarrollo económico responde a dos elementos principales: el primero, depende directamente de la capacidad de absorción de conocimientos por parte de las organizaciones, cuanto más empinada sea la curva de aprendizaje será mayor y mejor la forma como las organizaciones asimilan el conocimiento; la segunda depende el método y los conocimientos impartidos por las instituciones de educación, este último es realmente relevante, pues es sin duda alguna el medio a través del cual las empresas logran tener recurso humano ajustado a los retos impuesto por la economía.

La simbiosis existente entre la generación y absorción de conocimientos en el aparato productivo, otorga a las regiones una gran capaci- dad competitiva. El concepto capital intelectual hace referencia directa a ese segmento poblacional que posee competencias demandadas por las organizaciones, claro hablando desde el lenguaje practico. El presente documento se desarrolla en dos etapas la primera de ella es la revisión del sector productivo local, analizando su rasgos característicos y aportes a la economía de la región, y seguidamente el estudio de los rasgos del perfil demandado por el sector productivo.

\section{Aproximación teórica}

\section{Sector productivo de Cartagena de Indias}

Es de conocimiento general que el sector productivo de Cartagena de Indias recibe un gran beneficio de las actividades turísticas, siendo este uno de los principales renglones de la economía doméstica, sin embargo, el sector industrial ubicado en la zona de Mamonal también aporta al crecimiento de la ciudad; pero a la par de los sectores anteriormente mencionados se hallan otros cuyo aporte es menor pero no por ello menos significativo. La tabla 1, muestra algunas de las actividades económicas desarrolladas localmente y su aporte al PIB.

Tabla 1. Aporte de Cartagena al PIB comparación 2010 vs 2020

\begin{tabular}{lrr}
\hline \multicolumn{1}{c}{ ACTIVIDAD } & $\mathbf{2 0 1 0}$ & $\mathbf{2 0 2 0}$ \\
\hline Comercio, hoteles, restaurantes y transporte & $8,70 \%$ & $19,60 \%$ \\
Administración pública y defensa, salud y educación & $15,70 \%$ & $19,50 \%$ \\
Industrias manufactureras & $24,70 \%$ & $17,20 \%$ \\
Construcción & $7,60 \%$ & $11,80 \%$ \\
Agricultura, ganadería y pesca & $5,30 \%$ & $4,90 \%$ \\
Electricidad, gas y agua & $2,70 \%$ & $3,00 \%$ \\
Explotación de minas y canteras & $5,50 \%$ & $2,90 \%$ \\
Actividades financieras y de seguros & $11,10 \%$ & $2,70 \%$ \\
\hline Fuente: Elaboración de los autores a partir de Acosta-Ordoñez (2012) y CCC $(2021)$
\end{tabular}


Luego de observar la tabla 1, es interesante como algunos sectores mantuvieron una tendencia al crecimiento de su aporte al PIB mientras que otros como lo son: industrias manufactureras, agricultura, ganadería y pesca, y algunas actividades financieras y de seguros tuvieron una contracción; aunque los datos observados en los sectores que mostraron contracción en sus aportes al PIB son cautivador no son el objeto del presente estudio, por lo cual no se abordaran las razones que causaron dicho fenómeno.
De regreso al objeto de la investigación, es importante observar el crecimiento de las empresas que pertenecen al sector productivo local para lo cual se indignaron datos sobre la creación y sostenimientos de las empresas en el último año. De acuerdo con la cámara de comercio de Cartagena - CCC (2020), el sector productivo contaba con 29.834 empresas al finalizar 2020, distribuidas como muestra la tabla 2 .

Tabla 2. Empresas constituidas y existentes en 2020

\begin{tabular}{crr}
\hline & Existentes & Nuevas \\
\hline Micro & 26,791 & 6,869 \\
Pequeña & 2,199 & 31 \\
Mediana & 657 & 2 \\
Fuente: & Elaboración de los autores a partir de CCC (2021)
\end{tabular}

De acuerdo a los datos de CCC (2021), el sector productivo cartagenero está constituido principalmente por micro empresas y Pymes con un $89 \%$ y $7,40 \%$ respectivamente, la tendencia de crecimiento mostrada es de $99 \%$ de nuevas microempresas y tan solo $0,45 \%$ de pequeñas empresas.

Partiendo de los datos observados en las tablas 1 y 2, se puede deducir que la mayor fuerza productiva de la ciudad de Cartagena de Indias se encuentra en micro y pequeñas empresas de los sectores turístico, salud, educación e industrial y manufacturero.

\section{Perfil ocupacional de la fuerza productiva vinculada a las ciencias contables}

Cuando se pretende analizar cuál es perfil tecnológico y profesional demandado por las empresas es necesario observar las competencias más deseadas por los gerentes, tanto genéricas como específicas. En este aparte de la investigación se abordarán las competencias que deben poseer la fuerza productiva vinculada a las ciencias contables.

En primera instancia es relevante tener claridad del concepto de competencias. Bunk (1994) indica que desde la perspectiva laboral las competencias pueden ser entendidas como un cumulo de elementos como son: el conocimiento, la técnica, las actitudes y capacidades propias de los individuos y con lo cuál hacen frente a situaciones problémicas, aportando soluciones aterrizadas apoyadas en la creatividad.

Boterf (2001) coincide con Bunk (1994), al comprender las competencias como elementos que facultan a los individuos para actuar de la mejor manera en consecuencia al contexto, tomando la mejor decisión de elección de recursos propios o grupales para abordar situaciones complejas presente en las empresas.

Por su parte (Bustamante et al., 2002) vincula el concepto de incertidumbre haciendo referencia a situaciones presentes en los entornos caóticos donde los individuos deben tomar decisiones basadas en la razón.

Retomando la perspectiva de Bunk (1994); (González y Wagenaar, 2006) aseguran que las competencias implican la mixtura de conocimientos, habilidades, capacidades y valores. Lo anterior lleva a pensar que se está frente a un concepto cuya base es la integralidad. La vertiente propuesta por (Sarramona, 2007), contempla la integralidad puesto que indica que las competencias son la combinación de conocimientos, habilidades y actitudes que fa- 
cilitan actuar eficazmente ante situaciones. El concepto anterior esta sumergido en el enfoque práctico de la competencia.

En términos generales las competencias son el producto de varios factores: del ser como la ética, los valores y la conducta propia de los individuos; del saber cómo los conocimientos, habilidades y capacidades desarrolladas con el propósito de afrontar el entorno cambiante y los posibles problemas que este genere. Como ya se ha indicado existe distinto tipos de competencias desde las vinculadas con el ser hasta las que implican conocimiento, habilidades y destrezas (competencias de saber). Lo mencionado se ve apoyado por lo propuesto por Seltzer (2000), quien asegura que las competencias deben responder a una triple dimensión: 1) dimensión del saber lo cual significa los conocimientos generales y específicos que debe poseer los individuos, 2) la dimensión del hacer las cuales se relacionan con las habilidades necesarias para desempeñar un rol, 3) competencias del ser, se vinculan con la conduta y la ética de los individuos.

\section{Competencias relacionadas con el ser}

Rodríguez-Gómez (1998) afirma que las competencias son el resultado del comportamiento social afectivo, como las habilidades para la creación de conocimientos, la condición psicológica que inciden en la forma como se desempeñan los individuos al desarrollar una tarea.

Hassán (2009) aterriza las competencias del ser en el contexto de las ciencias contables indicando que este es un elemento esencial de la formación del capital humano, que le facilita la toma de decisiones para la solución de problemas donde la conducta debe ser intachable.

\section{Competencias del saber}

Para Mura y Coronel (2008) las competencias tienen componentes relacionados con la capacidad intelectual, los procesos de cognición que le faciliten entender y operar con ideas, conceptos y símbolos; dentro de estas competencias se encuentran las capacidades analíticas, las meta cognitivas.
Desde el enfoque de Villa y Poblete (2004) las competencias implican la puesta en marcha de conocimientos, habilidades, capacidades y valores que dan como resultado el correcto desempeño en ambientes caóticos y auténticos.

Por su parte, Medina (2009) indica que para formular competencias se debe tomar en consideración los conocimientos que el mercado laboral demanda, lo cuales se deben transmitir de forma que faciliten el desarrollo de capacidades para hacer frente a la realidad.

\section{Metodología}

El presente estudio es naturaleza cualitativa, que de acuerdo con Rueda (2007) es la conjunción de las distintas perspectivas cuyo propósito es comprender los elementos y las relaciones existente entre estos. Cerda (2002) otorga un carácter no cuántico a la investigación cualitativa, al indicar que esta se enfoca en identificar las características que describen perfectamente la relación de los individuos que forma la sociedad. En cuanto al tipo de investigación se aborda la investigación descriptiva, la cual comprende la descripción, la comprensión y el análisis de los fenómenos, mediante la indagación de los rasgos de los grupos de individuos o grupos de objetos Tamayo (2006).

La técnica de recolección de información es el análisis documental, lo que conlleva a utilizar fuentes secundarias que fundamentan el abordaje conceptual realizado. Es importante indicar que el estudio se realiza en dos fases la primera de ellas corresponde a la revisión literaria la cual da origen al presente artículo de revisión y la segunda fase corresponde a los resultados provenientes del trabajo de campo (recopilación de datos mediante una encuesta).

\section{Mapa de variables}

Para la construcción del mapa de variables de las competencias a desarrollar por los tecnólogos en contabilidad sistematizada se tomaron a consideración diversas fuentes como: la Federación Internacional de Contadores - IFAC (2019), (Roncancio et al., 2017), Montaño (2000); lo que permitió identificar las principales habilidades, valores y capacidades. 
Tabla 3. Mapa de variables de las competencias

\begin{tabular}{|c|c|}
\hline Variable & Dimensión \\
\hline \multirow{5}{*}{ 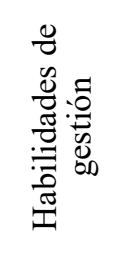 } & Capacidad de gestionar el talento humano \\
\hline & Capacidad de gestión del tiempo \\
\hline & Capacidad de adaptación al entorno \\
\hline & Capacidad de negociación \\
\hline & Capacidad para la toma de decisiones \\
\hline \multirow{7}{*}{$\frac{\mathscr{D}}{\stackrel{0}{0}} \frac{\pi}{\pi}$} & Integridad \\
\hline & Objetividad \\
\hline & Cumplimiento de normas \\
\hline & Honestidad \\
\hline & Actitud positiva \\
\hline & Ético \\
\hline & Sentido de pertenecía \\
\hline \multirow{6}{*}{ 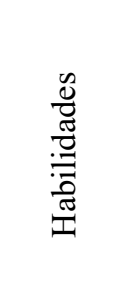 } & Liderazgo \\
\hline & Compromiso \\
\hline & Responsabilidad \\
\hline & Resolución de problemas \\
\hline & Disciplinado \\
\hline & Habilidades para gestión de herramientas TIC \\
\hline \multirow{4}{*}{ 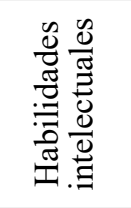 } & Lógica \\
\hline & Compresión \\
\hline & Creatividad \\
\hline & Pensamiento critico \\
\hline \multirow{13}{*}{ 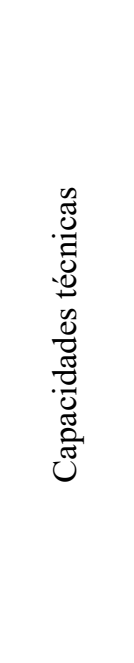 } & Conocimientos en materia financiera \\
\hline & Conocimientos tecnológicos \\
\hline & Conocimientos organizacionales \\
\hline & Conocimientos comerciales \\
\hline & Concomimientos en compras \\
\hline & Concomimientos en ventas \\
\hline & Manejo de sistemas contables \\
\hline & Conocimientos sobre costos \\
\hline & Conocimientos sobre Responsabilidad Social \\
\hline & Conocimientos sobre depreciaciones \\
\hline & Conocimientos sobre legislación fiscal \\
\hline & Conocimientos sobre ingresos y gastos \\
\hline & Conocimientos estadísticos para el análisis de \\
\hline
\end{tabular}

Fuente: IFAC (2019), (Roncancio et al., 2017), Montaño (2000)

\section{Resultados}

Basado en la revisión de los autores se puede vislumbrar un breve y conciso acercamiento al concepto de competencias, lo que lleva a comprender en términos generales que estas implican la integralidad de elementos de las diversas aristas de ser; más allá de conocimiento técnico especializados del área, es necesario formar a los futuros tecnólogos como individuos con la capa- cidad de amoldarse al entorno, con las capacidades para afrontar las situaciones propias de las organizaciones, siempre bajo el análisis crítico, con objetividad y responsabilidad social como guía de sus actuar.

En términos más específicos los tecnólogos en contabilidad deben ser formados para desarrollar principalmente habilidades, en distintos focos como son: las habilidades de gestión para la toma 
de decisiones frente a recursos y situaciones; habilidades blandas y transversales; habilidades del intelecto, dentro de las que caben el pensamiento crítico, la lógica, la comprensión y la creatividad, como se puede observar estas habilidades demandan un nivel más profundo de abstracción. Las capacidades técnicas involucran conocimientos especializados de las ciencias contables: en materia de finanzas, costos, legislación tributaria entre otros. Finalmente, los valores como base que soporta el proceso de formación, dentro de estos se pueden mencionar la integridad, la objetividad, la honestidad, el sentido de pertenencia y la Responsabilidad Social.

\section{Conclusiones}

La correcta formación y preparación del capital humano en tiempos de entornos convulsio- nados como los que afronta la humanidad actualmente, se convierte en un reto colosal para las Instituciones de Educación Superior, ya que el conocimiento transmitido a los estudiantes debe traspasar las fronteras de los especializado para convertirse en un proceso integral que transforme a técnicos, tecnólogos y profesionales en individuos con la capacidad de transformar la realidad, el entorno y los grupos sociales.

Desde el ámbito de las ciencias contables las exigencias son muy altas desde conocimientos técnicos hasta características del ser; la integralidad de los profesionales en ciencia contables contempla habilidades tecnológicas, dominios de sistemas tecnológicos contables y de gestión, capacidades intelectuales, como la lógica para resolución de problemas organizacionales y técnicas relacionadas con enfoque contable puro.

\section{Referencias}

Acosta-Ordoñez, K. (2012). Cartagena, entre el progreso industrial y el rezago social. Documentos de Trabajo Sobre Economía Regional y Urbana; No. 178.

Bunk, G. (1994). La transmisión de las competencias en la formación y perfeccionamiento profesionales de la RFA. Revista europea de formación profesional, (1), 8-14.

Bustamante, G., Torres, E., Marín, L., Gómez, J., \& Barrantes, E. (2002). El concepto de Competencia I. Una mirada interdisciplinar. Bogotá: Sociedad Colombiana de Pedagogía-Alejandría Libros.

CCC. (15 de mayo de 2021). Cartagena en cifras. www.cccartagena.org.co/wp-content/uploads/2021/03/2.Presentacion_-CTG-En-Cifras_-IV-Trimestre-de-2020.pdf

Cerda, G., H. (2002). Los elementos de la Investigación como reconocerlos, diseñarlos y construirlos. Editorial El Buho, 3ra edición, Bogotá D, C, Colombia.

González, J. \& Wagenaar, R. (2003). Tuning Educational Structures in Europe. Informe Final. Fase 1. Bilbao: Universidad de Deusto.

Hassán, J. O. C. (2009). Ética y responsabilidad social del contador: Perspectivas y tendencias frente a las IFRS. CAPIC REVIEW, (7), 83-94. https://dialnet.unirioja.es/descarga/articulo/3736704.pdf

IFAC (2019). An Illustrative Competency Framework for Accounting Technicians. www.ifac.org/system/files/publications/files/IFAC-AAT-An-Illustrative-Competency-Framework-for-Accounting-Technicians.pdf

Le Boterf, G. (2001). Ingeniería de las competencias. Barcelona: Epise.

Medina, A. (2009). Fundamentación de las competencias discentes y docentes (pp. 11-44). Formación y desarrollo de las competencias básicas. Madrid: Universitas.

Montaño, J. L. A. (2000). Capacidades no técnicas en el perfil profesional en contabilidad: las opiniones de docentes y profesionales. Revista española de financiación y contabilidad, 149-172. 
Mura, R., \& Coronel, J. (2008). La formación basada en las competencias en la materia práctica profesional. In XXX Simposio de Profesores de Práctica Profesional, Catamarca, Universidad Nacional de Catamarca, FCE y de administración.

Rodríguez-Gómez, Roberto. (1998). Conferencia mundial sobre educación superior. Revista mexicana de investigación educativa, ISSN 1405-6666, Vol. 4, Nº. 6, 1998.

Roncancio, A. D., Alvarado, G. E. M., \& Murcia, N. M. M. (2017). Las competencias en la formación del profesional contable: una revisión de las posturas institucionales y educativas en Colombia. Revista Facultad de Ciencias Económicas: Investigación y Reflexión, 25(2), 83-103.

Rueda, B., M. (2007). La investigación etnográfica y/o cualitativa y la enseñanza en la universidad. Revista mexicana de investigación educativa, 12(34), 1021-1041.

Sarramona, J. (2007). Las competencias profesionales del profesorado de secundaria. Estudios sobre educación, 12, 31-40.

Seltzer, J. C. (2000). Formando competencias. Economizarte. Buenos Aires, Argentina.

Sánchez, A. V., \& Ruiz, M. P. (2004). Practicum y evaluación de competencias. Profesorado. Revista de Curriculum y formación de profesorado, 8(2), 0.

Tamayo, M. (2004). El proceso de la investigación científica. Editorial Limusa. 